\title{
A new enemy is emerging in the fight against the SARS-CoV-2 pandemic
}

\section{Francesco Rodeghiero ${ }^{1}$ and Carlo L. Balduini ${ }^{2}$}

\author{
${ }^{1}$ Hematology Project Foundation, Vicenza and ${ }^{2}$ Fondazione Ferrata-Storti, Pavia, Italy \\ E-mail: CARLO L.BALDUINI - carlo.balduini@unipv.it
}

doi:10.3324/haematol.2021.279186

T he discovery of a new disease is always big news even more so when a new iatrogenic disorder emerges after the administration of a vaccine to millions of people during a global pandemic. This is precisely the case of vaccine-induced immune thrombotic thrombocytopenia (VITT) reported after vaccination against severe acute respiratory syndrome coronavirus 2 (SARS-CoV-2). VITT was first described in April 2021 by three independent groups in 39 people, 5 to 29 days after the first administration of the vaccine $\mathrm{ChAdOx} 1 \mathrm{nCoV}$ 19 (AstraZeneca), a recombinant chimpanzee adenoviral vector encoding the spike protein of SARS-CoV-2.1-3 Affected people were young or middle-aged adults, mostly women, who had acute onset of moderate to severe thrombocytopenia and thrombosis, often in unusual localizations such as cerebral venous sinus or portal, splanchnic, or hepatic veins. Other patients presented with venous thromboembolisms or acute arterial thrombosis. The disease was serious, with $40 \%$ mortality. Of note, nearly all patients had high levels of antibodies reacting to platelet factor 4 (PF4)-heparin complexes, as in heparin-induced thrombocytopenia, but they had not been previously exposed to this anticoagulant. This finding suggests a close correlation between VITT and autoimmune heparin-induced thrombocytopenia, which occurs in patients never exposed to heparin. The sequence of events leading to thrombosis did not emerge from these three pioneering studies. In particular, the nature of the polyanion macromolecule mimicking heparin remains uncertain. While the intact vaccine did not seem to be implicated, a pathogenic role of some spe- cific components, including the spike protein synthetized by the vaccine, could not be ruled out.

In this issue of Haematologica, two papers provide new information on VITT. The article by Althaus et al. ${ }^{4}$ describes the clinical and laboratory features of eight additional cases and significantly confirms the clinical and laboratory picture that has emerged from previous reports. Furthermore, this study provides new evidence of the pathogenesis of VITT by comparison of platelet activation induced by sera from patients with this condition with sera from subjects vaccinated without any clinical complications, as well as from patients with SARS$\mathrm{CoV}-2$. Finally, the authors report on the autopsy findings of two deceased VITT patients that revealed multidistrict thrombosis and showed kidney lesions similar to those of thrombotic microangiopathies.

The letter by Pomara et al. ${ }^{5}$ published in the same issue focuses on the results of a thorough post-mortem examination of two patients with typical VITT. The macroscopic picture was impressive revealing the extent of thrombosis which was much more widespread than initially revealed by imagining during life. The authors made extensive use of immunocytochemistry for the characterization of the affected organs and noted, among other interesting findings, platelet aggregates diffusely lining the endothelial layer of small and medium vessels. Pomara's paper, therefore, confirms the observation made by Althaus that VITT has this feature in common with thrombotic microangiopathies, confirming that generalized platelet activation plays a major role in this disorder.

The study of these additional ten new cases advances
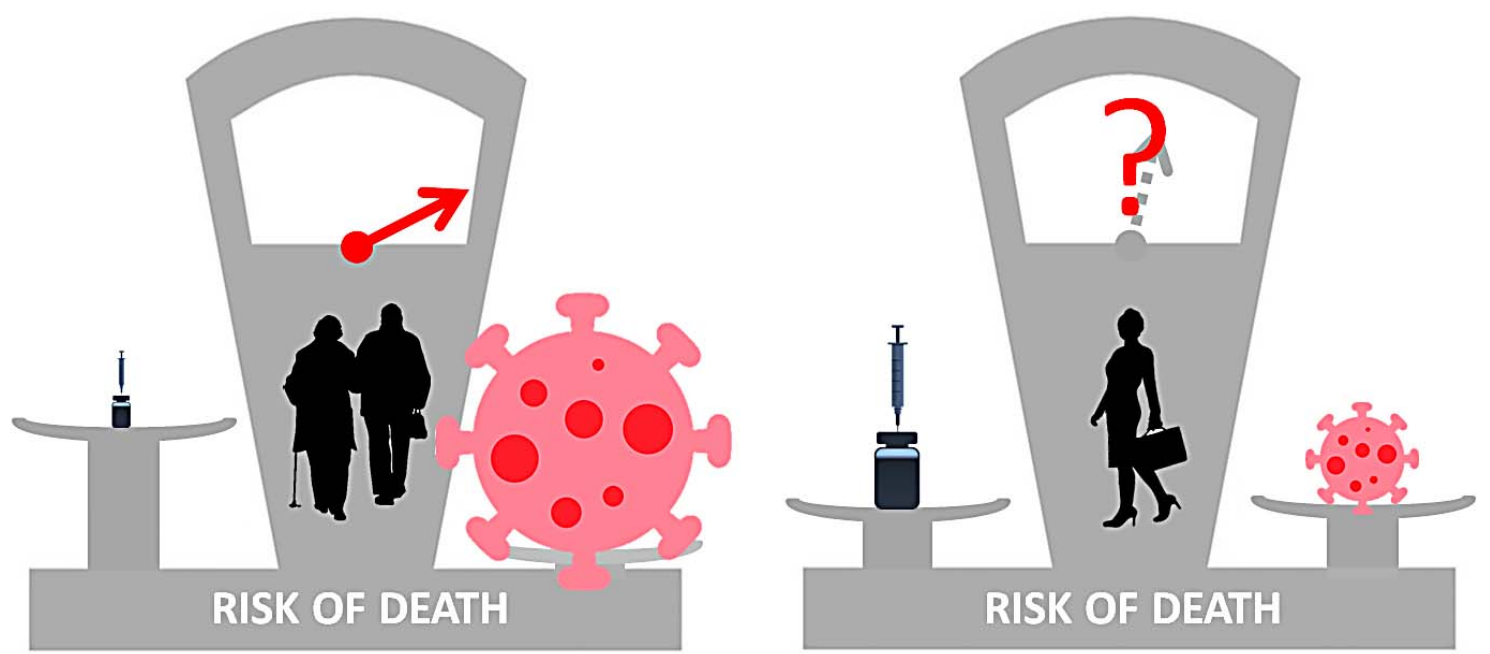

Figure 1. Of the 49 patients with vaccine-induced immune thrombotic thrombocytopenia reported to date, $79 \%$ were women and only $4 \%$ were over 60 years old. The risk of this disease, therefore, seems to be extremely low in old people who, on the other hand, have a high risk of death from SARS-CoV-2. The administration of adenoviral vaccines against SARS-CoV-2 is therefore highly advantageous in this category of subjects. Conversely, vaccine-induced immune thrombotic thrombocytopenia (VITT) seems to be more common in young or middle-aged adults, especially women, who instead have a risk of death from SARS-CoV-2 much lower and in some age categories even close to zero. Pending new data, these considerations have convinced some countries not to administer adenoviral vaccines to young subjects. 
the knowledge of VITT, which, however, still remains poorly defined because many questions await to be answered. First and foremost, how common is this complication after vaccination with $\mathrm{ChAdOx} 1 \mathrm{nCoV}-2$ ? At present, a total of only 49 cases have been described and the disease therefore appears exceptionally rare considering that millions of people have received this drug. However, VITT was only identified in April 2021 and, therefore, its diagnosis was impossible prior to this date and, hence, this complication may currently be greatly underreported.

As of 4 April 2021, a total of 169 cases of CVST and 53 cases of splanchnic vein thrombosis were reported to EudraVigilance in around 34 million people vaccinated in the EEA and UK (Europenan Medicine Agency). ${ }^{6}$ How many of these patients had VITT? Furthermore, the diagnosis of cerebral venous sinus and splanchnic vein thrombosis is not always easy, and we cannot exclude that their occurrence has so far been underestimated. We fear that the number of cases of VITT reported after the ChAdOx1 $\mathrm{nCoV}-19$ vaccine will augment in the coming months, as specific testing for anti PF4-polyanion complexes will be increasingly applied to clinically suspected cases.

Another important question is whether VITT is caused only by the ChAdOx1 $\mathrm{nCoV}-19$ vaccine or may be triggered by other adenoviral vaccines. The answer to this question was given by a paper ${ }^{7}$ published at the end of April 2021 that described 12 patients who developed a disease with the clinical and laboratory characteristics of VITT 5 to 26 days after vaccination with Ad26.COV2.S COVID-19 (Johnson \& Johnson/Janssen). This drug, too, uses a human adenoviral vector, which, however, differs in many respects from that used by the $\mathrm{ChAdOx} 1 \mathrm{nCoV}$ 19 vaccine. It is, therefore, possible that the risk of VITT is a common feature of drugs that use adenoviral vectors. It is important at this point to emphasize that adenoviral vectors are presently employed not only for vaccines against SARS-CoV-2 and EBOLA, ${ }^{8}$ but they are under investigation for vaccines against other infectious diseases, ${ }^{8}$ for cancer immunotherapy ${ }^{9}$ and for gene therapy of inherited disorders. ${ }^{10}$ Should it be shown that the risk of VITT is intrinsic to different adenovirus-based treatments, the risk-benefit ratio would be completely different if this approach is used to treat cancer or to prevent an infectious disease that has a mortality of about 1-2\% (and in some age groups very close to $0 \%$ ).

A final question is how to treat patients with VITT. Clinicians who have reported their experience in this field have generally suggested avoiding the use of heparin and platelet transfusions, given to correct the profound thrombocytopenia and associated cerebral hemorrhage. Instead, they believe that non-heparin anticoagulants and high-dose intravenous immunoglobulin could have some efficacy. These recommendations are mainly based on previous experience with heparininduced thrombocytopenia and heparin-induced autoimmune thrombocytopenia, and are currently only anecdotal experiences suggesting their use in VITT. A better understanding of the pathogenic mechanisms of VITT and prospective clinical studies are required to identify the best treatment for VITT, which is a rare or even very rare disease. Only large international collaborations will be able to ensure the provision of credible results in a short time. Furthermore, it has not been established if these treatments are also appropriate for any atypical thrombosis after vaccination, particularly in the absence of the specific laboratory features of VITT. A long way of laboratory and clinical research lies ahead.

\section{Disclosures}

No clonflicts of interest to disclose.

\section{References}

1. Greinacher A, Thiele T, Warkentin TE, Weisser K, Kyrle PA, Eichinger S. Thrombotic thrombocytopenia after ChAdOx1 nCov19 Vaccination. N Engl J Med. 2021;384(22):2092-2101.

2. Schultz NH, Sørvoll IH, Michelsen AE, et al. Thrombosis and thrombocytopenia after ChAdOx1 nCoV-19 vaccination. N Engl J Med. 2021;384(22):2124-2130.

3. Scully M, Singh D, Lown R, et al. Pathologic antibodies to platelet factor 4 after ChAdOx1 nCoV-19 vaccination. $N$ Engl J Med. 2021;384(23):2202-2211.

4. Althaus K, Möller P, Uzun G, et al. Antibody-mediated procoagulant platelets in SARS-CoV-2-vaccination associated immune thrombotic thrombocytopenia. Haematologica. 2021;106(8):2170-2179.

5. Pomara C, Sessa F, Ciaccio M, et al. Post-mortem findings in vaccineinduced Thrombotic Thombocytopenia. Haematologica. 2021;106 (8) $2291-2293$

6. European Medicines Agency. AstraZeneca's COVID-19 vaccine: EMA finds possible link to very rare cases of unusual blood clots with low blood platelets. https://www.ema.europa.eu/en/news/ astrazenecas-covid-19-vaccine-ema-finds-possible-link-very-rarecases-unusual-blood-clots-low-blood. Accessed 11 May 2021

7. See I, Su JR, Lale A, et al. US Case Reports of Cerebral Venous Sinus Thrombosis With thrombocytopenia after Ad26.COV2.S vaccination, March 2 to April 21, 2021. JAMA. 2021 Jun 22;325(24):24482456

8. Tomori $\mathrm{O}$, Kolawole MO. Ebola virus disease: current vaccine solutions. Curr Opin Immunol. 2021;71:27-33.

9. Tessarollo NG, Domingues ACM, Antunes F, et al. Nonreplicating adenoviral vectors: improving tropism and delivery of cancer gene therapy. Cancers (Basel). 2021;13(8):1863.

10. van Haasteren J, Li J, Scheideler OJ, Murthy N, Schaffer DV. The delivery challenge: fulfilling the promise of therapeutic genome editing. Nat Biotechnol. 2020;38(7):845-855. 